\section{PTH-060 CONVERSION OF EMR TO ESD: AN OPTION TO BE KEPT IN MIND!}

Adolfo Parra-Blanco*, Shiv Budihal, Krish Ragunath. Nottingham Digestive Diseases Centre, University of Nottingham, UK NIHR BRC at Nottingham University Hospitals and University of Nottingham, UK, Nottingham, UK

\subsection{6/gutjnl-2019-BSGAbstracts.85}

Introduction Endoscopic Mucosal Resection (EMR) is a procedure applied for the treatment of most colorectal polyps. Endoscopic Submucosal Dissection (ESD) has the significant advantage of providing en bloc specimens, but it's more technically demanding, time-consuming, and the learning curve is steep.

Conversion to snare resection during difficult colorectal ESD has been described, and factors related to this have been identified. However, conversion from EMR to ESD has not been described.

Methods A 53 year old male patient was referred for rectal bleeding. A $30 \mathrm{~mm}$ Paris 0-Ip, Kudo IV polyp was identified on a colonoscopy, and was referred to the SPECC MDT. Polypectomy was recommended.

A flexible signmoidoscopy was performed (Olympus Lucera Spectrum Elite 290 endoscopy system, PCF260AZI colonoscope). There was a large polyp at the lower and mid rectum, with estimated size $5 \mathrm{~cm}$. Initially it looked pedunculated, but on close inspection it was in fact sessile, although the base apparently was not very broad, The lesion was bulky and a piecemeal EMR was anticipated to be difficult, but it was still considered feasible, and arrangements for an ESD hadn't been done.

The base of the polyp was injected with a solution of Gelofusin and indigo carmine; $20 \mathrm{~mm}$ and $25 \mathrm{~mm}$ Olympus Snare Master snares were applied to attempt resection of the lesion. An ERBE VIO 200D unit was utilised. Underwater technique was utilised to encircle the whole lesion with the snare, but it was not possible. Only two small pieces of the polyp were resected. When a larger piece of the polyp was snared, it felt firm and the lesion was not cut despite using not only forced coagulation current, but also endocut, in several intervals of 5 seconds.

A decision was made to switch to ESD in the patient's best interest. No sedation had been given, only Entonox. The patient understood the indication for ESD, and agreed with proceeding.

A scissor-type knife (SB knife Junior, Sumitomo) was applied for the ESD. A soft straight distal attachment (Olympus) was utilised for the EMR, and it was maintained for the ESD.

Large vessels were pre-coagulated with soft coagulation before cutting them. The resection took $30 \mathrm{~min}$ for the EMR attempts, and 57 minutes for the ESD. There were no complications. The post ESD site was sutured with 4 clips. The patient was discharged home after the procedure.

Results Histopathological analysis revealed a tubulovillous adenoma with low grade dysplasia.

Conclusions ESD with a SB knife can be used as a free hand technique in cases of large pedunculated and sessile lesions in which EMR is not resolutive. The importance of adequate polyp assessment in planning the best treatment strategy for colorectal polyps cannot be overstated.

\section{PTH-061 IMPROVING BOWEL PREP AND COLONOSCOPY UNDERSTANDING WITH AN EDUCATIONAL VIDEO}

${ }^{1}$ Thomas Archer*, ${ }^{1}$ Nikhil Lal, ${ }^{2}$ Claire Emms, ${ }^{3}$ Andrew Baxter, ${ }^{2}$ Keith Dear, ${ }^{3}$ Adolfo Parra Blanco, ${ }^{1}$ Mo Thoufeeq. ${ }^{1}$ Sheffield Teaching Hospital, Sheffield, UK; ${ }^{2}$ Chesterfield Royal Hospital, Chesterfield, United Kingdon; ${ }^{3}$ Nottingham University Hospital, Nottingham, UK

\subsection{6/gutjnl-2019-BSGAbstracts.86}

Introduction Bowel preparation (BP) is key to high quality colonoscopy. Up to $20 \%$ of colonoscopies may be limited by inadequate BP. Good quality BP is shown to increase Adenoma Detection Rate, and reduces procedure length. Understanding and adherence to instructions is associated with quality of BP. The use of visual aids can lead to improved BP. We aim to introduce an educational video as a quality improvement project and assess its acceptability and impact on patients' understanding of how to take BP for colonoscopy.

Method A video was created in collaboration with the Nottingham Trent University graphics department. The video was reviewed by a patient involvement group and doctors. Patients referred for colonoscopy were included. An initial survey of patients was conducted to assess their understanding of colonoscopy and BP. Subsequently, a group of patients referred for a colonoscopy watched the educational video and completed questionnaires. Outcome data from their endoscopy reports was also accessed to assess adequacy of BP.

Results A preliminary survey of patients demonstrated that 10/ 47 patients rated their understanding of a colonoscopy procedure or how to take BP as $5 / 10$ or less on a visual analogue scale (VAS). $38 \%$ of these patients had inadequate BP, compared with $8 \%$ of the patients whose score $>5 / 10$. Subsequently 32 patients (male to female ratio 1:1) were recruited and were shown the educational video.

The mean age was 64 and the range was 49-83. 22 patients rated the acceptability and how easy it was to follow the video as 10/10. 3 rated the acceptability and how easy it was to follow the video < 5/10. $24(75 \%)$ patients indicated that the video improved their understanding of what a colonoscopy involved, 1 indicating a decrease in understanding and the remaining 7 patients indicating no change in understanding. 24 of patients who viewed the video had a colonoscopy of whom 1 patient had inadequate BP, 4 had fair BP and 19 had good or excellent BP.1

\begin{tabular}{ll} 
Abstract PTH-061 Table 1 & \\
\hline Understanding of how to take BP following & Number of patients \\
viewing the video & \\
\hline Improved & $22 / 32$ \\
Remained the same & $9 / 32$ \\
Decreased & $1 / 32$
\end{tabular}

Conclusion The majority of patients in this cohort found the video both acceptable and easy to follow and improved understanding of what a colonoscopy involved and how to take BP for the procedure. Although the sample was small and wasn't matched to controls, the outcome in terms of adequacy of BP was excellent. The data indicates that an educational video would be acceptable and could improve both understanding of 